\title{
The NEA High Priority Nuclear Data Request List for future needs
}

\author{
A.J.M. Plompen ${ }^{1, a}$, T. Fukahori ${ }^{2}$, H. Henriksson ${ }^{3, b}$, A. Ignatyuk ${ }^{4}$, T. Iwasaki ${ }^{5}$, G. Manturov ${ }^{4}$, R.D. McKnight ${ }^{6}$, \\ G. Rimpault ${ }^{7}$, and D.L. Smith ${ }^{6}$ \\ ${ }^{1}$ European Commission, Joint Research Centre, Institute for Reference Materials and Measurements, 2440 Geel, Belgium \\ 2 Japanese Atomic Energy Agency, Nuclear Data Center, Tokai-mura, Naka-gun, Ibaraki-ken 319-1195, Japan \\ ${ }^{3}$ Organisation for Economic Co-operation and Development, Nuclear Energy Agency, 92130 Issy-les-Moulineaux, France \\ 4 Institute of Physics Power Engineering, 249020 Obninsk, Kaluga Region, Russia \\ 5 Department of Quantum Science and Energy Engineering, Tohoku University, Sendai 980-8579, Japan \\ 6 Department of Energy, Argonne National Laboratory, Argonne, IL 60439, USA \\ 7 Commissariat à l'Énergie Atomique, DEN/DER/SPRC/LEPh, 13108 Saint-Paul-lez-Durance, France
}

\begin{abstract}
The Nuclear Energy Agency (NEA) of the Organisation for Economic Co-operation and Development (OECD) established the Working Party on Evaluation Co-operation (WPEC) to promote exchange of information on nuclear data activities by its members. One of the WPEC objectives is to assess needs for nuclear data improvements and to stimulate initiatives to fulfill these. The High Priority Request List (HPRL) was established in pursuit of this objective. The HPRL project aims at identifying accuracy targets for the improvement of nuclear data, primarily for application in the nuclear industry. The list therefore provides a guide for those planning new measurements, nuclear model calculations and data evaluation programmes.

Since 2004, the HPRL has undergone a major overhaul to better guarantee timeliness and adequate documentation of requests. A new webpage was developed at the NEA to facilitate submission of new requests, and to allow easy inspection. Submitted requests are first reviewed by external referees, before being accepted on the list. Requests are divided into high priority requests, where a quantitative justification is provided and general requests for which the justification is qualitative. The relevance of requests for nuclear energy applications is ensured through periodic reviews by an expert group of the NEA WPEC, involving Europe (JEFF), Japan (JENDL), the United States (ENDF), Russia (BROND) and China (CENDL). The new procedures for submission, review and adoption of requests were recently validated with a first set of entries. Examples of recent updates to the HPRL project are presented. The requirements for providing new requests are also presented, as well as how to justify needs for new nuclear data. Data users around the world are encouraged to formulate their concerns about nuclear data and submit them to the HPRL. The HPRL is maintained by the NEA Data Bank and can be consulted at the following address: http://www.nea.fr/html/dbdata/hprl/.
\end{abstract}

\section{Introduction}

The Nuclear Energy Agency (NEA) is a specialised agency within the Organisation for Economic Co-operation and Development (OECD), an intergovernmental organisation of industrialised countries, based in Paris, France. The NEA Data Bank works within an international network of several data centres in charge of the compilation and dissemination of basic nuclear data. The NEA's nuclear data evaluation co-operation activities proceed through the Working Party on Evaluation Co-operation (WPEC) and involve evaluation projects in the following regions: Japan (JENDL), United States (ENDF), Western Europe (JEFF), and non-OECD member countries (BROND, CENDL, and FENDL). The participation of nonOECD member countries in these evaluation projects is channelled through the Nuclear Data Section of the International Atomic Energy Agency (IAEA). The Working Party is established to promote the exchange of information on nuclear data evaluations, measurements, nuclear model calculations, validation, and related topics, and to provide a framework for co-operative activities between the participating projects.

\footnotetext{
${ }^{a}$ Presenting author

${ }^{b}$ Contact person, e-mail: Henriksson@nea. fr
}

The Working Party assesses needs for nuclear data improvements and addresses those needs by initiating joint evaluation and/or measurement efforts. The Nuclear Data High Priority Request List, HPRL, is a compilation of the most important nuclear data requirements for nuclear energy applications, both current and future systems (e.g., transmutation, Generation-IV nuclear reactors or fusion applications). One of the subgroups ${ }^{1}$ of WPEC is responsible for drawing up the HPRL, which is designed to provide guidance to those managing the measurement and evaluation programmes on the requirements for improved nuclear data for applications, primarily but not exclusively, in nuclear power technology.

The first High Priority Nuclear Data Request List was produced in the early 1980 s by the NEA Committee on Reactor Physics (NEACRP), and the NEA Nuclear Data Committee (NEANDC). The NEANDC reviewed progress in meeting the requirements and commented on the status of the available data and the feasibility of meeting the requirements through the WPEC and the Working Party on Measurement Activities (WPMA, 1996-1998). Around 2000, the HPRL was under criticism from policy makers that it was 1) very long, that

\footnotetext{
${ }^{1}$ Subgroup-C; the present authors are the current members of subgroup-C and the NEA contact person (HH).
} 


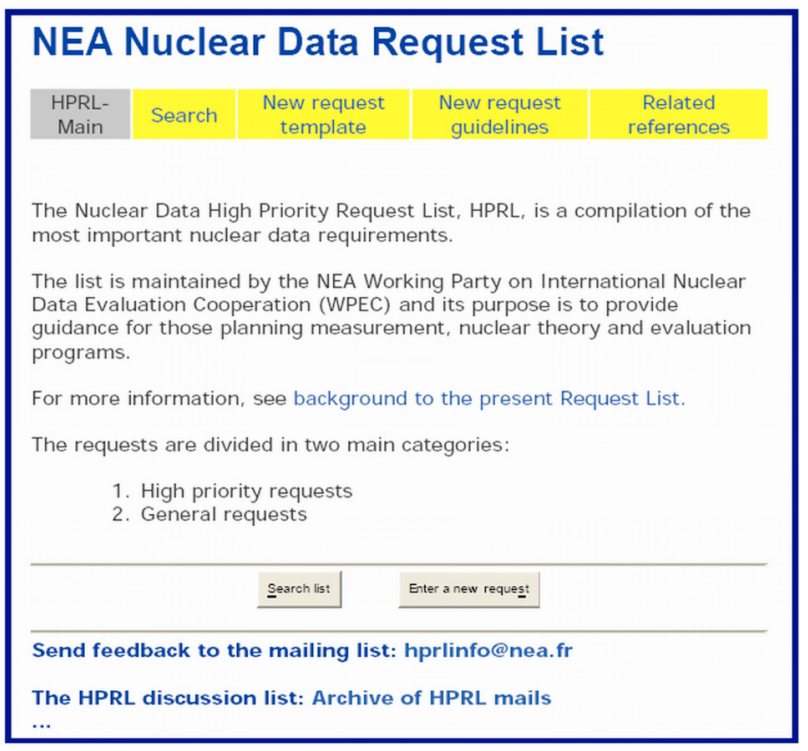

Fig. 1. The HPRL webpage, http://www.nea.fr/html/dbdata/hprl/.

2) not all of its entries concerned issues that were of current interest, and that 3 ) the information concerning requests was very terse, contact points being hard to identify.

\section{The new High Priority Request List}

Since 2004, the HPRL has undergone a major overhaul to better guarantee timeliness and adequate documentation of requests.

\subsection{The webpage}

A new webpage (fig. 1) was developed at the NEA to facilitate submission of new requests, and to allow easy inspection. The new webpage ensures that a minimum amount of information concerning a new request is provided and clearly specifies the requester and how to contact him or her. The webpage is largely self-explanatory and at the same time provides extensive guidance to users. The entry page shown in figure 1 introduces the HPRL, refers to the background document and provides the essential links for the users. The main menubar consisting of the "HPRL-Main", "Search", "New request template", "New request guidlines", and "Related references" fields is accessible on each page. The list may be consulted through the "Search"-field and new requests may be entered via the field "New request template". An extensive explanatory document is accessible through the "New request guidelines" link. Additional references of general relevance to the HPRL are also accessible through the main menu-bar.

\subsection{Submitting a new request}

Data users around the world are encouraged to formulate their concerns about nuclear data and submit them to the HPRL. Figure 2 summarises the fields of the request form that

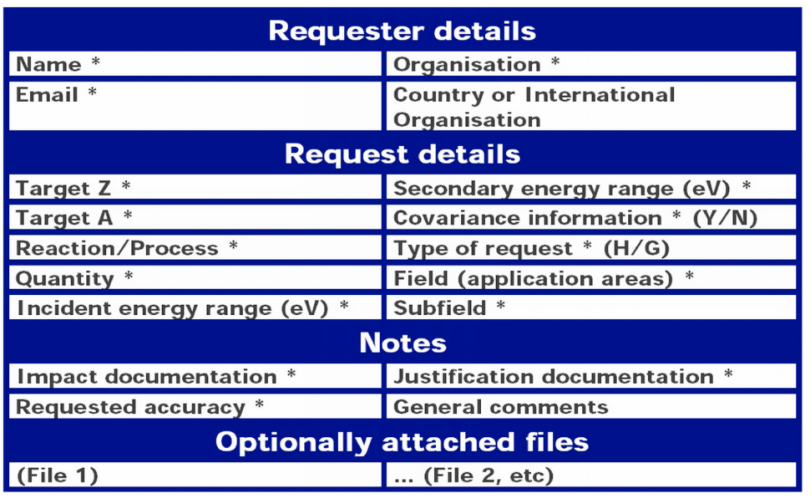

Fig. 2. The fields of the HPRL request form.

have to be entered upon application of a new request. Fields marked with an asterisk are obligatory. The additional level of information, that is required for the new HPRL and was not available in the previous editions, concerns the first box Requester details - and the third box - Notes - which asks for impact documentation, requested accuracy, justification documentation and general comments. To facilitate preparation of a request, the relevant parts of the "textitnew request guidelines"-document will pop-up when hovering over certain fields in the on-line request template. In case of the fields "reaction/process", "quantity" and "field (application areas)" drop down menus are available, while for the "Covariance information" and "Type of request" fields the user indicates yes or no for the first and high priority or general request for the second field.

As is clear from figure 2, the applicant indicates whether a request is considered of high priority or of general importance. An important difference with respect to earlier editions of the HPRL, concerns the fields for justification and for impact documentation. The justification field is the heart of the request. It should summarise concisely the case behind the request justifying why a new effort is required. The impact documentation field should indicate which applications stand to benefit once the request is fulfilled. Since - with minor modifications - the text entered in these fields appears online via the search form, concise and well formulated summaries should be provided. The use of references and the uploading of support documents is encouraged to further clarify these summaries.

In the case of a high priority request stating a particular target accuracy, a quantitative justification must be provided. Ideally, a sensitivity study links the target accuracy for the requested data to the target accuracy for the parameter of concern to the application. For such a request appropriate impact documentation should support the claim for high priority. For general requests the importance of the justification and impact fields is relaxed allowing for more scientifically oriented pursuits for data improvement. The link to a nuclear energy application must, however, remain evident.

\subsection{Adoption and follow-up of a request}

Once a request is submitted it appears in the list as "to be checked" indicating that it is up for review. The review is 
carried out by Subgroup-C with the possible assistance of external referees who will iterate with the requester if further clarification is required. Following this negotiation phase the request is either adopted or rejected. At any time, external parties can provide feedback through an e-mail link that appears on the page with the details of the request. Such information will be checked and, if appropriate, added to the description of the request along with the comments already provided during the review process.

The review serves to guarantee timeliness and importance of the HPRL entries. It is therefore not only carried out for a new request but is repeated periodically to eliminate from the list what is outdated as a result of new developments or of new insights.

It must be stressed that the seeming rigidity of the procedure and forms is indeed only apparent. More than one requester may support a single entry allowing for justification and impact documentation with views from different angles. Requests may be updated or upgraded as additional information comes along. More than two files may be uploaded if this is more convenient. In all cases, the NEA contact person can be contacted to handle aspects that may not be dealt with using the online request form. Interested parties may interact with any member of the subgroup or through the HPRL mailing list: hprl@nea.fr.

Once a request is adopted members of Subgroup-C will encourage follow-up by advertising it in their respective communities.

\section{Building up the new list}

\subsection{A clean slate}

The new High Priority Request List for nuclear data started with a clean slate. The new procedures for submission, review and adoption of requests were validated on the basis of a first set of entries and currently eight entries feature on the list. Further new requests are anticipated to emerge from active subgroups of WPEC. Similarly, it is anticipated that validation and evaluation efforts of the most recent versions of the ENDF, JEFF, JENDL, BROND, CENDL and IAEA libraries and associated special sub-libraries will lead to new contributions. It is stressed that suggestions may be advanced by any person from any organisation as long as they fall within the scope of the NEA and WPEC and are deemed justified.

\subsection{Adopted requests}

Here a number of examples of new requests are presented to illustrate the above and to provide a stimulus for follow up.

\subsubsection{High priority requests}

The ${ }^{16} \mathbf{O}(\mathbf{n}, \boldsymbol{\alpha}){ }^{13} \mathbf{C}$ reaction. WPEC expert group "Subgroup 22" on "Nuclear data for improved LEU-LWR reactivity predictions" identified the ${ }^{16} \mathrm{O}(\mathrm{n}, \alpha){ }^{13} \mathrm{C}$ reaction as one of several possible sources of criticality under prediction for light water reactor benchmarks [1]. The sensitivity coefficient ${ }^{2}$ of $-3.5 \mathrm{pcm} / \%$ combined with a $30 \%$ uncertainty for the data in the range from 2.5 to $10 \mathrm{MeV}$ amount to an uncertainty for $k_{\text {eff }}$ of $100 \mathrm{pcm}$. Clearly, justification and impact are well established for this entry. Additional justification is derived from the contribution of this reaction to helium production in fuel pins and clads ( $7 \%$ unc. from a $25 \%$ contribution) and the relevance of this reaction for neutron source calibrations through the manganese-sulfate bath technique.

Follow-up activity for this request was initiated at the Institute for Reference Materials and Measurements in the form of a new measurement campaign by G. Giorginis and co-workers [2].

\section{Prompt gamma-production by fission of ${ }^{239} \mathrm{Pu}$ and} ${ }^{235} \mathrm{U}$. Two high priority requests concern improved data for prompt gamma-production by fission of ${ }^{239} \mathrm{Pu}$ and ${ }^{235} \mathrm{U}$ from the thermal to the fast neutron energy region $(0-10 \mathrm{MeV})$ with a desired accuracy better than $7.5 \%$. The issue is of relevance to estimates of gamma-ray heating in non-fuel zones for four fast reactor systems under study in the GenerationIV initiative and for the Jules Horowitz Reactor (RJH) to be established at CEA Cadarache. The requested accuracy for the data corresponds to the requested accuracy for the heating. From studies of recent benchmarks it is clear that the target accuracy is not met. Forty percent of the total gamma-ray energy release results from prompt decay of fission products. Furthermore, no comprehensive analytic expressions exist and Hauser-Feshbach model calculations are involved and presently lack sufficient knowledge to warrant a solution of the problem. New measurements would be needed to guide new evaluation efforts. Present evaluations are based on measurements from the seventies. Several references are provided on the webpage of the HPRL and in the accompanying uploaded support document (e.g., [3]).

The neutron capture cross section of hafnium. For the capture cross section of hafnium a $4 \%$ accuracy is requested in the energy range from 0.5 to $5000 \mathrm{eV}$, to resolve underestimations of the reactivity worth in benchmarks involving the AZUR zero-power reactors of CEA. The underestimation is attributed to the overestimation of the capture cross section. No recent library solves the problem and also a recent reevaluation of the available data was insufficient. New accurate measurements are therefore required. Hafnium is used to control the fission process in nuclear power reactors and studies to improve the reactivity worth problem are of interest to nuclear industry [4].

Neutron elastic scattering by deuterium. The double differential elastic scattering cross section of neutrons on deuterium in the range from 0.1 to $1 \mathrm{MeV}$ is of importance to (advanced) CANDU reactors. Different representations of the energy-angle probability distributions in various recent libraries cause differences of 1) $1000 \mathrm{pcm}$ in simulations of

\footnotetext{
$21 \mathrm{pcm}=10^{-5}$.
} 
critical experiments with uranyl fluoride solutions in heavy water, 2) biases for heavy-water coolant void reactivity in ZED-2 experiments. Available differential scattering experimental data are between 25 and 50 years old and are sparse and inconsistent, particularly at backward angles. Data scatter several standard deviations from the evaluated library values. Empirical sensitivity studies established the energy range of interest. A TSUNAMI sensitivity analysis shows a sensitivity coefficient of 34 (34\% change for $1 \%$ change of the elastic cross section) for the coolant void reactivity. The current methodology does not allow a further quantification of the accuracy requirement of the angle dependence. The request is well documented including five references (e.g., [5]). Followup activities in the form of improved differential experiments are being investigated at several laboratories.

\subsubsection{General requests}

The ${ }^{28} \mathbf{S i}(\mathbf{n}, \mathbf{x})^{27} \mathrm{Al}$ reaction. Silicon carbide $(\mathrm{SiC})$ is a potential very low activation structural material for a fusion power reactor. The concentration of the ${ }^{26} \mathrm{Al}$ radionuclide $\left(T_{1 / 2}=7.2 \cdot 10^{5} \mathrm{y}\right)$ determines whether the decommissioned fusion blanket qualifies for recycling. ${ }^{26} \mathrm{Al}$ results from the above-mentioned reaction and the ${ }^{27} \mathrm{Al}(\mathrm{n}, 2 \mathrm{n})^{26} \mathrm{Al}$ reaction in a two step process. According to the most recent evaluation for this cross section the estimated concentration limits for $\mathrm{Si}$ are a factor 10 higher than earlier estimates for a neutron wall load of $5 \mathrm{MW} / \mathrm{m}^{2}$ [6]. Thus, $\mathrm{SiC}$ would qualify as a truly low-activation material, however, no experimental verification of this important assertion is available, whence the present request.

The ${ }^{233} U(n, \gamma){ }^{234} U$ reaction, request-6. ${ }^{233} U$ is the primary fissile isotopes of the thorium-uranium $(\mathrm{Th} / \mathrm{U})$ fuel cycle. The most important cross section for this cycle is that for the ${ }^{233} \mathrm{U}(\mathrm{n}, \mathrm{f})$ reaction. The capture cross section is of importance for estimates of the ${ }^{233} \mathrm{U}$ inventory in $\mathrm{Th} / \mathrm{U}$ reactors and for the inventory estimates for the heavier isotopes that evolve from it. Profil and Profil-2 experiments in the Phenix reactor at CEA Marcoule have shown an underestimation of the capture cross section by $9 \%$ [7]. The request is for an improved accuracy of better than $9 \%$ in the energy region from $10 \mathrm{keV}$ to $1 \mathrm{MeV}$ and impacts fast reactors using $\mathrm{Th} / \mathrm{U}$ as a fuel. The available data are insufficient to improve the cross section. New measurements are encouraged, but owing to the experimental difficulties involved, the best approach may be to take account of the integral results in a new evaluation.

The ${ }^{56} \mathrm{Fe}(\mathrm{n}, \mathrm{xn})$ reaction. A recent global pre-equilibrium analysis from 7 to $200 \mathrm{MeV}$ by Koning and Duijvestijn noted the absence of experimental double differential $(n, x n)$ data in this energy region which is of interest to accelerator driven systems. To guide model development a result on a single nucleus is requested with an accuracy of $30 \%$. Fe is proposed as a ubiquitous structural material. This request is an example of a generic scientific issue that concerns transport calculations of energetic neutrons in and near spallation neutron sources.

The ${ }^{233} \mathrm{U} \alpha$ and $\eta$ parameters, request-9. This second request concerning ${ }^{233} \mathrm{U}$ emerges from investigations by CNRS in France for Molten Salten Reactors using the Th/U fuel cycle. Of critical importance to such reactors is the stability of the fissile isotope $\left({ }^{233} \mathrm{U}\right)$ inventory which is a delicate balance of production and destruction terms, named the regeneration gain (RG). A sensitivity study of the RG shows two important contributions: the mean number of neutrons produced per fission of ${ }^{233} \mathrm{U}$ and the capture cross section. Important quantities are therefore the fission to capture cross section ratio $\alpha$ and the fission neutron production to capture cross section ratio $\eta$. As molten salt reators are essentially thermal systems the energy range of interest is from thermal to several $\mathrm{keV}$. The current uncertainty on $R^{3}$ is $4200 \mathrm{pcm}$ and the target accuracy is requested to be $2000 \mathrm{pcm}$, which involves a simultaneous reduction of the capture cross section uncertainty from $10 \%$ to $5 \%$ and of $\bar{v}$ from $1 \%$ to $0.5 \%$.

\section{Conclusion}

The new High Priority Request List for nuclear data has been presented with emphasis on the new features compared to earlier editions. The practical aspects are greatly facilitated through a new webpage, which guides requesters in providing the additional information that is needed. The webpage facilitates searching the request list and provides a greatly improved level of documentation. A review process warrants timeliness and the priority of requests. Finally, the currently available requests were presented.

\section{References}

1. A. Courcelle (co-ordinator) et al., Report NEA/WPEC-22, NEA No. 6199, ISBN 92-64-02317-8, available online http://www.nea.fr/html/science/wpec/volume22/volume22.pdf, OECD Nuclear Energy Agency (2006).

2. G. Giorginis et al. (these proceedings).

3. G. Rimpault, Proc. Workshop on Nuclear Data Needs for Generation IV, April 2005, Antwerp, Belgium, ISBN 981-256-830-1 (World Scientific, 2006), p. 18.

4. G. Noguère et al., JEFDOC-1077, Joint Evaluated Fission and Fusion file project, OECD NEA (2005) (unpublished).

5. K.S. Kozier et al., Proc. of the PHYSOR-2006 ANS Topical Meeting, Vancouver, Sep. 2006; K.S. Kozier et al. (these proceedings).

6. E.T. Cheng, J. Nucl. Mat. 258-263, 1767 (1998).

7. J. Tommasi et al., Nucl. Sci. Eng. 154, 119 (2006). 